\title{
Error Analysis of Calibration Materials on Dual-Energy Mammography
}

\author{
Xuanqin Mou and Xi Chen \\ Institute of Image Processing and Pattern Recognition, Xi'an Jiaotong University \\ Xi'an, Shaanxi, 710049, China \\ xqmou@mail.xjtu.edu.cn, chenxi@mailst.xjtu.edu.cn
}

\begin{abstract}
Dual-energy mammography can suppress the contrast between adipose and glandular tissues and improve the detectability of microcalcifications (MCs). In clinical dual-energy mammography, imaging object is human breast, while in calibration measurements, only phantoms of breast-tissue-equivalent material can be used. The composition and density differences between calibration materials and human breast bring the differences of linear attenuation coefficient which lead to the calculation errors in dual-energy imaging. In this paper, the magnitude of MC thickness error from calibration materials has been analyzed using a first-order propagation of error analysis. This analysis shows that the thickness error from calibration materials ranges from dozens to thousands of microns which can not be ignored when carrying out dual-energy calculations. The evaluation of several popular phantoms shows that it is of great importance to adopt the phantom materials approaching human breast most.
\end{abstract}

Keywords: dual-energy, mammography, calibration materials, error analysis.

\section{Introduction}

Microcalcifications (MCs) are the principal indicator of breast cancer. Thus the visualization and detection of MCs in mammography play a crucial role in reducing the rate of mortality of breast cancer. MCs are composed mainly of calcium compounds. Although MCs are small in size, almost below $1 \mathrm{~mm}$, they have greater x-ray attenuation than the surrounding breast tissue. This makes MCs relatively apparent on homogeneous soft-tissue backgrounds. However, visualization of MCs may be obscured in mammograms by overlapping tissue structures.

In dual-energy digital mammography, high- and low-energy images are acquired and synthesized to suppress the contrast between adipose and glandular tissues, improve the detectability of MCs and calculate the thickness of the MC. However, quantitative dual-energy imaging can be influenced by many factors in practical application, such as the selection of high- and low-energy spectra, scatter, quantum noise, DQE (detection quantum efficiency) of detectors and the choice of calibration polynomials, all of which decrease the calculation precise 
and cause quantitative information inaccurate. Research work in dual-energy imaging has mainly focused on the selection and optimization of high- and lowenergy spectra [1], scatter corrections [2], analysis of detectors [3] and selection of inverse-map functions [4.

In clinical dual-energy mammography, the imaging object is human breast, while in calibration measurements, only phantoms of breast-tissue-equivalent material can be used. Also, the elemental composition ratio of human breast presents a wide range [5].The composition and density differences between the phantom materials and human breast bring the differences of linear attenuation coefficients which lead to the calculation error in dual-energy imaging. However, such error hasn't been accounted for in the published literatures on dual-energy imaging till now. In this paper, we assess the magnitude of the calculation error introduced by calibration materials using an error propagation analysis. Simulated experiments verify the magnitude of such error in dual-energy mammography.

\section{Theory}

\subsection{Physical Model of Dual-Energy Imaging}

In mammography, the breast is compressed to the thickness $T$ which is to be composed of adipose tissue (thickness $t_{a}$ ), glandular tissue (thickness $t_{g}$ ) and MC (thickness $t_{c}$ ). As the total breast thickness $T$ is known and the contribution of MCs to the total breast thickness can be ignored, the three unknowns of $t_{a}, t_{g}$, $t_{c}$ can be expressed as two: glandular ratio $g=t_{g} /\left(t_{a}+t_{g}\right) \cong t_{g} / T$ and $\mathrm{MC}$ thickness $t_{c}$.

If $P_{0}(E)$ is the incident x-ray photon fluence of energy $E$ and $P(E)$ is the transmitted fluence, then:

$$
P(E)=P_{0}(E) \exp \left[-\mu_{a}(E) T-g\left(\mu_{g}(E)-\mu_{a}(E)\right) T-\mu_{c}(E) t_{c}\right]
$$

$\mu_{a}(E), \mu_{g}(E)$ and $\mu_{c}(E)$ are the linear attenuation coefficients of adipose tissue, glandular tissue and MCs, respectively.

In dual-energy imaging calculations, a reference signal $I_{r}$ is needed to increase the dynamic range of the logarithmic intensity value. Now we define the exposure data $f$ as the log-value of ratio of the transmitted exposure $I$ and reference signal $I_{r}$. The low- and high-energy log-value $f_{l}\left(t_{c}, g\right), f_{h}\left(t_{c}, g\right)$ are measured independently using $\mathrm{x}$-ray beams of different $\mathrm{kVps}$ :

$$
\left\{\begin{aligned}
f_{l}= & \ln \left(I_{r l}\right) \\
& -\ln \left\{\int P_{0 l}(E) \exp \left[-\mu_{a}(E) T-g\left(\mu_{g}(E)-\mu_{a}(E)\right) T-\mu_{c}(E) t_{c}\right] Q(E) d E\right\} \\
f_{h}= & \ln \left(I_{r h}\right) \\
& -\ln \left\{\int P_{0 h}(E) \exp \left[-\mu_{a}(E) T-g\left(\mu_{g}(E)-\mu_{a}(E)\right) T-\mu_{c}(E) t_{c}\right] Q(E) d E\right\}
\end{aligned}\right.
$$

$Q(E)$ is the detector response.

The goal of dual-energy mammography is to convert the log-value functions $f_{l}\left(t_{c}, g\right)$ and $f_{h}\left(t_{c}, g\right)$ to MC thickness $t_{c}\left(f_{l}, f_{h}\right)$ and $g\left(f_{l}, f_{h}\right)$. Polynomials are 
often used to describe the relationship between $\left(f_{l}, f_{h}\right)$ and $\left(t_{c}, g\right)$ in diagnostic dual-energy imaging calculations. In this paper, cubic polynomials are used to describe the inverse-map functions:

$$
\left\{\begin{array}{l}
t_{c}=k_{c 0}+k_{c 1} f_{l}+k_{c 2} f_{h}+k_{c 3} f_{l}^{2}+k_{c 4} f_{h}^{2}++k_{c 5} f_{l} f_{h}+k_{c 6} f_{l}^{3}+k_{c 7} f_{h}^{3} \\
g=k_{g 0}+k_{g 1} f_{l}+k_{g 2} f_{h}+k_{g 3} f_{l}^{2}+k_{g 4} f_{h}^{2}++k_{g 5} f_{l} f_{h}+k_{g 6} f_{l}^{3}+k_{g 7} f_{h}^{3}
\end{array}\right.
$$

Coefficients $k_{i j}(i=c, g ; j=0,1, \cdots, 7)$ are approximated numerically by least-squares technique according to calibration data.

\subsection{Calculation Errors from Calibration Materials}

After necessary correction procedures, the image data $\left(f_{l}, f_{h}\right)$ are free of scatter and noise; systemic calculation bias $\Delta t_{c}$ of MC thickness still exists in dualenergy imaging. $\Delta t_{c}$ is composed of two portions: first, it is an approximation that MC thickness and glandular ratio represented by polynomials. Substituting the $\log$-values $\left(f_{l}, f_{h}\right)$ into the calibrated polynomials to calculate MC thickness is an interpolation of calibration data intrinsically which results in fitting error $\Delta t_{c p}$. Secondly, materials for calibration always differ from materials imaged. The differences of linear attenuation coefficient between calibration materials and imaged human breast lead to calibration material error $\Delta t_{c c}$ of $\mathrm{MC}$ thickness.

Isolated calibration material error $\Delta t_{c c}$ could not be obtained directly from inverse-map polynomials Eq, 3 , but can be deduced from dual-energy physical model Eq,2. Physically, the differences between calibration materials and imaged human breast can be represented in Eq2 where $f_{l}$ and $f_{h}$ are log-values of imaged human breast and $\mu_{a}(E), \mu_{g}(E)$ and $\mu_{c}(E)$ are linear attenuation coefficients of calibration materials. At the energy of $E$, the partial derivative of $t_{c}$ with respect to $\mu_{a}(E)$ can be deduced from dual-energy physical model Eq2 using differential method for implicit function:

$$
\frac{\partial t_{c}}{\partial \mu_{a}(E)}=\frac{-\frac{\partial f_{l}}{\partial \mu_{a}(E)} \times S_{g}+\frac{\partial f_{h}}{\partial \mu_{a}(E)} \times R_{g}}{R_{c} \times S_{g}-R_{g} \times S_{c}}
$$

where:

$$
R_{c}=\partial f_{l} / \partial t_{c} \quad R_{g}=\partial f_{l} / \partial g \quad S_{c}=\partial f_{h} / \partial t_{c} \quad S_{g}=\partial f_{h} / \partial g
$$

The partial derivatives of $t_{c}$ with respect to $\mu_{g}(E)$ and $\mu_{c}(E)$ can be obtained in the same way. As we know, the first-order terms of a Taylor's series expansion can be used for error estimation. And then, the magnitude of calibration material error in MC thickness $\Delta t_{c c}$ can be approximated using the first-order terms of a Taylor's series expansion of $t_{c}$ in terms of the variables $\mu_{i}(E)(i=g, a, c)$ :

$$
\Delta t_{c c} \approx \sum_{E} \frac{\partial t_{c}}{\partial \mu_{g}(E)} \times \Delta \mu_{g}(E)+\sum_{E} \frac{\partial t_{c}}{\partial \mu_{a}(E)} \times \Delta \mu_{a}(E)+\sum_{E} \frac{\partial t_{c}}{\partial \mu_{c}(E)} \times \Delta \mu_{c}(E)
$$

where $\Delta \mu_{i}(E)(i=g, a, c)$ are the differences of the linear attenuation coefficient between calibration materials and imaged human breast. 


\section{Materials and Methods}

The simulation conditions which are in accordance with the clinical equipments are as same as those in papers [2] 4] of Kappadath.

The x-ray spectra used for low- and high-energy measurements were $25 \mathrm{kVp}$ and $50 \mathrm{kVp}$. The spectra data were taken from Handbook of Mammographic $X$-ray Spectra [6] with resolution of $1 \mathrm{keV}$.

The detector consists of a CsI:Tl converter layer coupled with an aSi:H flatpanel detector. All photons transmitted through the imaged object were assumed to be absorbed completely in the perfectly efficient converter layer [7.

Hammerstein [5] has determined the elemental compositions of glandular and adipose tissues in a human breast. The composition per weight of $\mathrm{H}, \mathrm{N}$, and $\mathrm{P}$ seems to be well determined; however, those of $\mathrm{C}$ and $\mathrm{O}$ present a wide range of possible values. Table 1 lists two extreme compositions 1 and 2, one general composition 3. In this paper, the composition per weight of $\mathrm{C}$ and $\mathrm{O}$ refer to composition 3 without specific notification. The densities equal to $0.93 \mathrm{~g} / \mathrm{cm}^{3}$ for adipose tissue and $1.04 \mathrm{~g} / \mathrm{cm}^{3}$ for glandular tissue.

Table 1. Elemental compositions (percentage per weight) of $\mathrm{C}$ and $\mathrm{O}$ for glandular and adipose tissues

\begin{tabular}{ccccccc}
\hline & \multicolumn{2}{c}{ Composition 1 } & \multicolumn{2}{c}{ Composition 2 } & \multicolumn{2}{c}{ Composition 3 } \\
\cline { 2 - 7 } Element & Adipose & Glandular & Adipose & Glandular & Adipose & Glandular \\
\hline $\mathrm{C}$ & 68.1 & 30.5 & 51.3 & 10.8 & 61.9 & 18.4 \\
$\mathrm{O}$ & 18.9 & 55.6 & 35.7 & 75.3 & 25.1 & 67.7 \\
\hline
\end{tabular}

Phantom materials were used for calibration. In the simple way, polyethylene $\left(\mathrm{CH}_{2}\right)$ was commonly used for adipose phantom material, PMMA $\left(\mathrm{C}_{5} \mathrm{H}_{8} \mathrm{O}_{2}\right)$ and acrylic $\left(\mathrm{C}_{3} \mathrm{H}_{4} \mathrm{O}_{2}\right)$ were often used for glandular phantom material. Breast-tissueequivalent materials can be available commercially. For example, breast-tissueequivalent materials from CIRS (Computerized Imaging Reference Systems, Inc., Norfolk, VA, USA) are based on Hammerstein and those from RMI (Radiation Measurements Inc., Madison, WI, USA) are based on White 8]. The elemental compositions of phantom materials 9] and human breast are listed in Table 2 .

In this paper, MCs were assumed to be composed mainly of calcium oxalate $\left(\mathrm{CaC}_{2} \mathrm{O}_{4}\right)$. The density is $2.20 \mathrm{~g} / \mathrm{cm}^{3}$. Aluminum was used to calibrate MCs.

Mass attenuation coefficients of the materials in this paper were computed by XCOM software from NIST [10].

The calibration data consisted of 30 different combinations of MC thicknesses and glandular ratios. Glandular ratio was in five steps 0\%, 25\%, 50\%, 75\% and $100 \%$ at a fixed total breast thickness $5 \mathrm{~cm}$; MC thickness was in six steps 0 , 380, 760, 1140, 1520, $1900 \mu \mathrm{m}$. The reference signal $I_{r}$ was measured through $50 \%$ glandular ratio of $5 \mathrm{~cm}$ breast phantom. 
Table 2. Elemental compositions (percentage per weight) of phantom materials and human breast

\begin{tabular}{cccccccccccc}
\hline & Density & \multicolumn{1}{c}{ Elemental compositions } \\
\cline { 3 - 13 } Materials & $\left(\mathrm{g} / \mathrm{cm}^{3}\right)$ & $\mathrm{H}$ & $\mathrm{C}$ & $\mathrm{N}$ & $\mathrm{O}$ & $\mathrm{F}$ & $\mathrm{Cl}$ & $\mathrm{Ca}$ & $\mathrm{P}$ & $\mathrm{Al}$ \\
\hline For adipose & & & 1 & & & & & & & \\
\hline Polyethylene & 0.93 & 14.4 & 85.6 & - & - & - & - & - & - & - \\
CIRS & 0.924 & 11.8 & 76.0 & 1.2 & 9.8 & - & 1.2 & - & - & - \\
RMI & 0.92 & 8.4 & 69.1 & 2.4 & 16.9 & 3.1 & 0.1 & - & - & - \\
Human & 0.93 & 11.2 & 61.9 & 1.7 & 25.1 & - & - & - & 0.1 & - \\
\hline For glandular & & & & & & & & & & & \\
\hline PMMA & 1.19 & 8.0 & 60.0 & - & 32.0 & - & - & - & - & - \\
Acrylic & 1.19 & 5.6 & 50.0 & - & 44.4 & - & - & - & - & - \\
CIRS & 1.04 & 10.9 & 70.2 & 1.2 & 12.5 & - & 1.1 & 0.6 & - & 3.5 \\
RMI & 0.99 & 8.4 & 68.0 & 2.3 & 18.9 & - & 0.1 & 2.3 & - & - \\
Human & 1.04 & 10.2 & 18.4 & 3.2 & 67.7 & - & - & - & 0.5 & - \\
\hline
\end{tabular}

\section{Results}

\subsection{Calibration Material Errors}

After calibration, substitute the log-values $\left(f_{l}, f_{h}\right)$ into the calibrated polynomials and calculate MC thickness $t_{c}^{\prime}$, the systemic calculation bias is $\Delta t_{c}=$ $t_{c}^{\prime}-t_{c}$. When calibration materials are identical to human breast, the calibration material error $\Delta t_{c c}$ is zero and the fitting error $\Delta t_{c p}$ equals $\Delta t_{c}$. When calibration materials are phantom materials, $\Delta t_{c}$ consists of $\Delta t_{c p}$ and $\Delta t_{c c}, \Delta t_{c c}$ can be calculated according to Eq 5

Tables 3 lists $\Delta t_{c c}, \Delta t_{c p}$ and $\Delta t_{c}$ when different calibration materials used where $\mathrm{MC}$ is $250 \mu \mathrm{m}$ and glandular ratio is $70 \%$. In the first three rows, only one calibration material is different from the composition of human breast. Then we can get the MC thickness errors where $1 \%$ linear attenuation coefficient differences exist. In the last three rows, phantom materials were adopted.

In Table 3. fitting error is only $-2 \mu \mathrm{m}$. The calibration material errors are $-33 \%(-83 \mu \mathrm{m}),-138 \%(-346 \mu \mathrm{m})$ and $1.2 \%(3 \mu \mathrm{m})$ when $1 \%$ linear attenuation coefficient differences exist between the calibration materials for adipose, glandular, MC and human beast, respectively. The errors coming from the calibration materials for glandular and adipose tissues are more than errors from $\mathrm{MC}$ calibration material.

If CIRS phantoms and aluminum were adopted as calibration materials, the calibration errors of MC thickness are 169\%(423 $\mu \mathrm{m})$. CIRS phantoms are made based on the human breast composition; however, composition differences still exist. In the energy range $10 \mathrm{keV}-50 \mathrm{keV}$, the average linear attenuation coefficient differences are $0.95 \%$ between CIRS adipose phantom and breast adipose, $1.14 \%$ between CIRS glandular phantom and breast glandular, $0.95 \%$ between aluminum and $\mathrm{CaC}_{2} \mathrm{O}_{4}$. 
Table 3. MC thickness errors using various calibration materials $\left(t_{c}=250 \mu \mathrm{m}, g=\right.$ $70 \%$ )

\begin{tabular}{cccccc}
\hline \multicolumn{3}{c}{ Calibration materials } & \multicolumn{3}{c}{ MC thickness errors $(\mu m)$} \\
\hline Adipose & Glandular & Microcalcification & $\Delta t_{c c}$ & $\Delta t_{c p}$ & $\Delta t_{c}$ \\
\hline $0.99 \mathrm{HM}$ & $\mathrm{HM}^{1}$ & $\mathrm{CaC}_{2} \mathrm{O}_{4}$ & -83 & -2 & -84 \\
$\mathrm{HM}$ & $0.99 \mathrm{HM}$ & $\mathrm{CaC}_{2} \mathrm{O}_{4}$ & -346 & -2 & -310 \\
$\mathrm{HM}$ & $\mathrm{HM}$ & $0.99 \mathrm{CaC}_{2} \mathrm{O}_{4}$ & 3 & -2 & -0.3 \\
$\mathrm{CIRS}^{2}$ & $\mathrm{CIRS}$ & $\mathrm{Al}$ & 423 & -2 & 404 \\
$\mathrm{RMI}^{3}$ & $\mathrm{RMI}$ & $\mathrm{Al}$ & -9419 & -2 & -9163 \\
Polyethylene & PMMA & $\mathrm{Al}$ & 2066 & -2 & 2134 \\
\hline
\end{tabular}

${ }^{1}$ human breast composition determined by Hammerstein

2 phantom material produced by CIRS

3 phantom material produced by RMI

If RMI phantoms, polyethylene and PMMA were used for calibration, the tremendous calibration material errors make the calculated MC thickness insignificant.

\subsection{Calibration Material Errors of Different Breast Compositions}

The breast composition of different individuals is variable while the calibration materials are always fixed when dual-energy imaging. The variations in breast composition of different individuals also bring about the MC thickness errors. Table 4 lists the errors of the three compositions listed in Table 1] Calibration materials are CIRS phantoms and aluminum. The MC thickness errors are far apart when calibration materials fixed and breast compositions changing.

Table 4. Calibration material errors of different breast compositions $\left(t_{c}=250 \mu \mathrm{m}\right.$, $g=70 \%)$

\begin{tabular}{cccc}
\hline & \multicolumn{3}{c}{ MC thickness errors $(\mu m)$} \\
\cline { 2 - 4 } Breast compositions & $\Delta t_{c c}$ & $\Delta t_{c p}$ & $\Delta t_{c}$ \\
\hline Composition 1 & -182 & -6 & -182 \\
Composition 2 & 912 & -6 & 905 \\
Composition 3 & 423 & -6 & 404 \\
\hline
\end{tabular}

\section{Discussion}

In this paper, we analyze the MC thickness calculation errors brought by attenuation differences between calibration materials and human breast.

Comparing $\Delta t_{c c}$ and $\Delta t_{c p}$, the fitting error $\Delta t_{c p}$ is relatively small. The calibration material error $\Delta t_{c c}$ occupies the dominant position in the systemic thickness bias $\Delta t_{c}$, it may be thousands of microns sometimes which makes the calculated MC thickness insignificant. 
As can be seen in Table 3, it is impractical to use RMI phantoms or polyethylene, PMMA as calibration materials since they may bring about huge calibration material errors. In contrast, CIRS phantom materials are made based on human breast and approaching the compositions of human breast nearest in the published literatures. However, it will bring about 160\% calibration material errors. Figure 1 presents the calibration material percent errors when glandular ratio ranges from $0 \%$ to $100 \%, \mathrm{MC}$ thickness ranges from $0 \mu \mathrm{m}$ to $1000 \mu \mathrm{m}$. The errors are almost positive which means that calibration using CIRS phantoms and aluminum will make the calculated $\mathrm{MC}$ thicker and will not cause the $\mathrm{MC}$ invisible. These calibration material errors may be ignored when dual-energy mammography is just used for the purpose to enhance the detectability of MCs. The calibration material percent error increases as the glandular ratio increasing, and decreases as the MC thickness increasing.

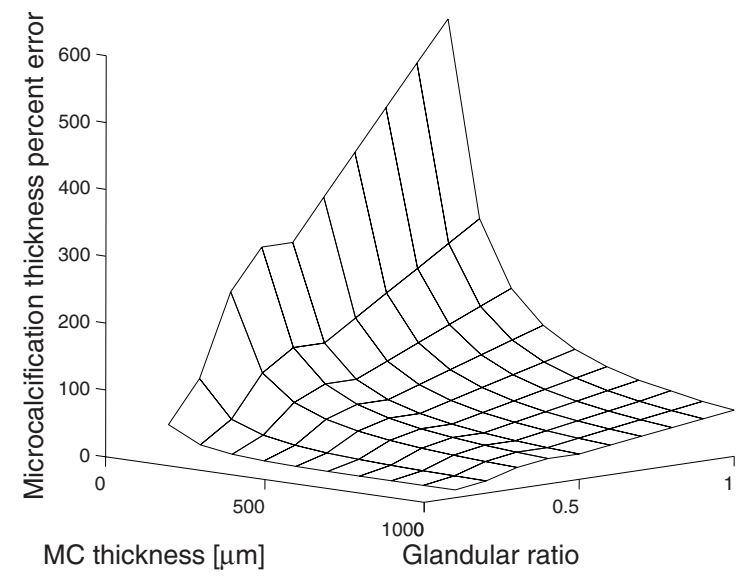

Fig. 1. MC thickness percent errors from calibration materials CIRS phantoms and aluminum

\section{Conclusion}

Since the attenuation characteristics of calibration materials are not exactly equal to those of human breast and the breast composition of different individuals is variable, MC thickness errors from calibration materials are unavoidable. From the point of imaging physics, this paper investigates the magnitude of calibration material error, ranging from dozens to thousands of microns. The errors from calibration materials can not be ignored when carrying out dual-energy calculations. The simple phantom materials, such as PMMA or polyethylene, are not suitable for calibration in dual-energy mammography in clinic. CIRS phantoms are the better choice.

In this paper, we analyze the physical model of dual-energy imaging and derive the expression that quantifies the calibration material error. Calculating 
based on the physical model is an effective way to analyze errors in dual-energy imaging.

Acknowledgments. The project is partially supported by National Nature Science Fund of China (No.60472004 and No.60551003 ), and the fund of the Ministry of Education of China (No. 106143).

\section{References}

1. Johns, P.C., Yaffe, M.J.: Theoretical Optimization of Dual-energy X-ray Imaging with Application to Mammography. Med. Phys. 13, 289-296 (1985)

2. Kappadath, S.C., Shaw, C.C.: Dual-energy Digital Mammography for Calcification Imaging: Scatter and Nonuniformity Corrections. Med. Phys. 32, 3395-3408 (2005)

3. Guillemaud, R., Robert-Coutant, C., Darboux, M., Gagein, J.J., Dinten, J.M.: Evaluation of Dual-energy Radiography with a Digital X-ray Detector. Proc. SPIE 4320, 469-478 (2001)

4. Kappadath, S.C., Shaw, C.C.: Dual-energy Digital mammography: Calibration and Inverse-mapping Techniques to Estimate Calcification Thickness and Glandulartissue Ratio. Med. Phys. 30, 1110-1117 (2003)

5. Hammerstein, G.R., Miller, D.W., White, D.R., Masterson, M.E., Woodard, H.Q., Laughlin, J.S.: Absorbed Radiation Dose in Mammography. Radiology 130, 485491 (1979)

6. Fewell, T.R., Shuping, R.E.: Handbook of Mammographic X-ray Spectra. HEW Publication (FDA), Washington. D.C. (1978)

7. Lemacks, M.R., Kappadath, S.C., Shaw, C.C., Liu, X., Whitman, G.: A Dual-energy Subtraction Technique for Microcalcification Imaging in Digital Mammography-A Signal-to-noise Analysis. Med. Phys. 29, 1739-1751 (2002)

8. White, D.R.: The Formulation of Tissue Substitute Materials Using Basic Interaction Data. Phys. Med. Biol. 22, 889-899 (1977)

9. Byng, J.W., Mainprize, J.G., Yaffey, M.J.: X-ray Characterization of Breast Phantom Materials. Phys. Med. Biol. 43, 1367-1377 (1998)

10. XCOM, http://physics.nist.gov/PhysRefdata/Xcom/Text/XCOM.html 